\title{
SOME EFFECTS OF NOISE ON HUMAN BEHAVIOR
}

\author{
NATHAN H. AZRIN
}

Anna State Hospital, 1 Anna, Illinois

Past studies of the effects of high-intensity noise on man have presented a picture of conflicting results that are often contrary to expectations. Intuitively, we might expect intense noise to disrupt performance. However, Kryter (16), in an extensive review of the literature, concluded that intense noise does not affect performance and, further, that "nearly all industrial and laboratory experiments which report that noise adversely affects work output are open to criticism because of poor experimentation and uncontrolled factors. (By 'work' is meant any mental, and motor tasks not involving communication by speech.) On the other hand, experiments carried out with proper control of all pertinent factors reveal that steady or expected noises do not adversely affect psychomotor activity to any significant extent (p. 22)." Berrien (4), in an independent review of this same subject, similarly concludes that there have been few satisfactory studies "in spite of uncritical acceptance of the assumption that noise because it is annoying must be harmful (p. 158). Unlike Kryter, however, Berrien concluded that there is sufficient evidence to believe that output and speed of work are somewhat affected.

Regardless of the final conclusion, it would appear that, at best, noise produces behavioral changes which are slight or transitory in duration $(5,8,11,14,21)$. Much more typical is the almost complete absence of behavioral change as a function of the presence of intense noise $(2,9,10,13,15,18,19,20,28,29)$. These results have seemed especially surprising in view of the strong subjective reactions which often accompany intense noise and the ease with which statements of annoyance can be obtained (17).

However, intuitive analysis also indicates that noise can occasionally be desirable. A deafening noise, for example, may actually be welcome if it indicates that a jet engine, previously silent because of mechanical difficulties, is now functioning again. None of the foregoing studies differentiates the effects of the stimulus, noise, in terms of its use as a discriminative stimulus or a response-contingent stimulus. Yet, such use might contribute to a better understanding of how noise affects behavior. In this investigation the effects of noise upon performance were studied when the presence or absence of noise had fixed temporal relationships (Experiment I) to neither the responses or the reinforcement; (Experiment II) to reinforcement; and (Experiment III) to responses.

1 This investigation was conducted at the Human Engineering Laboratory, Aberdeen Proving Ground. 


\section{GENERAL PROCEDURE}

Subjects

The subjects were 80 soldiers, volunteers, age 17-25. Each subject participated for 1 - 3 days, about 6 hours per day inter rupted only midday by a lunch period. Communication between subjects was minimized by selecting them from a unit which had almost $100 \%$ turnover about every 2 weeks. Audiograms were obtained from many of the subjects before and after participation to detect possible hearing losses.

\section{Apparatus and Procedure}

The method used in the present study was essentially the same as that developed by Holland (12) for measuring observing behavior. The subject was seated in a closed and sound-attenuating room. In front of the subject was a meter-needle, the deflection of which constituted the target. This target was presented every 3 minutes. The subject was instructed to release a switch located beneath the meter as soon as he saw a deflection of the needle. The meter-needle returned to its normal position as soon as the subject released the switch and not before then.

The room was darkened and the needle-deflection could not possibly be seen unless the subject pressed a button which served to illuminate the needle for 0.1 second after each depression, regardless of the duration of the depression. Accordingly, the faster the subject depressed the button, the more illumination he produced and the greater the opportunity he had for reacting quickly to the target when it was presented. These illuminating or observing responses were recorded automatically on a cumulative recorder.

Each subject was exposed to a 3-minute, fixed-interval schedule of reinforcement, the reinforcer being the target, until the pattern of observing responses showed a stable temporal discrimination for at least 30 minutes. The fineness of the temporal discrimination was evaluated in terms of the extent of the pause (no observing responses) following each reinforcement.

Manipulanda

During the experiment the manipulandum was changed. Initially, a manipulandum (button) was used which required very little force (about 15 grams) and a very small excursion (about 2 millimeters) in accordance with the procedures found suitable for animal conditioning (6). With this "light button," the rate of response usually was maintained at several responses per second with little or no scallops during prolonged exposure to a fixed-interval schedule of reinforcement. Later, however, the force requirement of the button was increased to several hundred grams, the over-all rate decreased, and scalloping took place much earlier in training. The observing responses appear to have had such a high unconditioned rate that the heavier button was needed to reduce this rate to a manageable operant level. Accordingly, the heavier button was substituted and used for the major part of the investigation. 2

Noise was delivered through two enclosed speaker systems located about 2 feet from each side of the subject's chair. Unless specified otherwise, white noise was. used with equal ( \pm 3 decibels) intensity from $80-8000$ cycles per second. The noise

\footnotetext{
2 Acknowledgment is made to Dr. E.C. Weiss for his suggestion concerning the modification of the manipulandum.
} 
was produced by a continuous loop of tape playing through a tape recorder, amplified by a 50-watt amplifier, and delivered to the two loud-speakers. The ambient noise level in the experimental chamber was about 55 decibels with the speakers disconnected. All programming and recording were performed automatically in a control room, two rooms from the experimental chamber.

\section{Instructions}

During the initial portion of the investigation, instructions were minimal, the subject being told only that he was in a target-detection study and that he was to react as quickly as possible to the target by releasing a designated switch. Frequently, the instructions were made more complete in order to hasten the learning process.

Under the more explicit instructions, the subjects were told that illumination would be produced if the illuminating button were depressed, and that the targets would be coming on a few minutes apart. A preliminary practice period followed, during which the subject was also given his target-detection time. Under these more explicit instructions, stable fixed-interval behavior usually emerged within 1 hour; under minimal instruction, the emergence of such behavior required from 3 to 12 hours. Noise produced no differential effects which could be related to these differing types of instructions.

\section{EXPERIMENT I: BEHAVIORAL EF FECTS OF NONCONTINGENT NOISE}

\section{Intense Noise}

This experiment was a study of the effects of noncontingent noise on fixed-interval performance, that is, presentation of the noise was governed neither by $\underline{\mathbf{S}} \mathbf{s}$ responses or by the reinforcements, but purely according to E. Figure 1 shows the effect of intense noncontingent noise. This figure presents a typical cumulative record of the observing responses of one of the ten subjects who were exposed to alternating periods of noise and quiet.

The first QUIET period (55 decibels) represents the stable pattern of observing responses after several hours of conditioning on a 3-minute, fixed-interval schedule of reinforcement. It can be seen that virtually no responses occurred during the first 2 minutes of each 3-minute interval (e.g., at A), after which time responses were made at a high rate. This rate terminates with the presentation and detection of the target at the end of the 3-minute interval. This pattern was characteristic of the performance of all $\underline{S}^{\prime} s$ during fixed-interval conditioning. The absence of response following reinforcement, and the high rate of response immediately preceding reinforcement, defines $\underline{S^{\prime}} \mathbf{s}$ temporal discrimination of the 3-minute interval between reinforcements.

B marks the first introduction of noise. This noise period continued for $\mathbf{3 0}$ minutes for this one $\underline{S}$, but varied from 15 - 90 minutes for the other nine $\underline{S}^{\prime} s$. The immediate effect of the noise was the elimination of the temporal discrimination, as evidenced by the fact that responses were made without pause during the entire 3-minute interval beginning at $B$. The temporal discrimination gradually returned, however. At $\mathbf{C}$ a distinct pause followed reinforcement, and this pause grew progressively longer during the succeeding intervals until the pattern of responding during noisepresentation closely approximated the pattern during the previous QUIET period. This disruption at the onset of intense (95-110 decibels) noise lasted from 3 15 minutes for the other nine $\underline{S}^{\prime} s$, with virtually complete recovery in each case. 


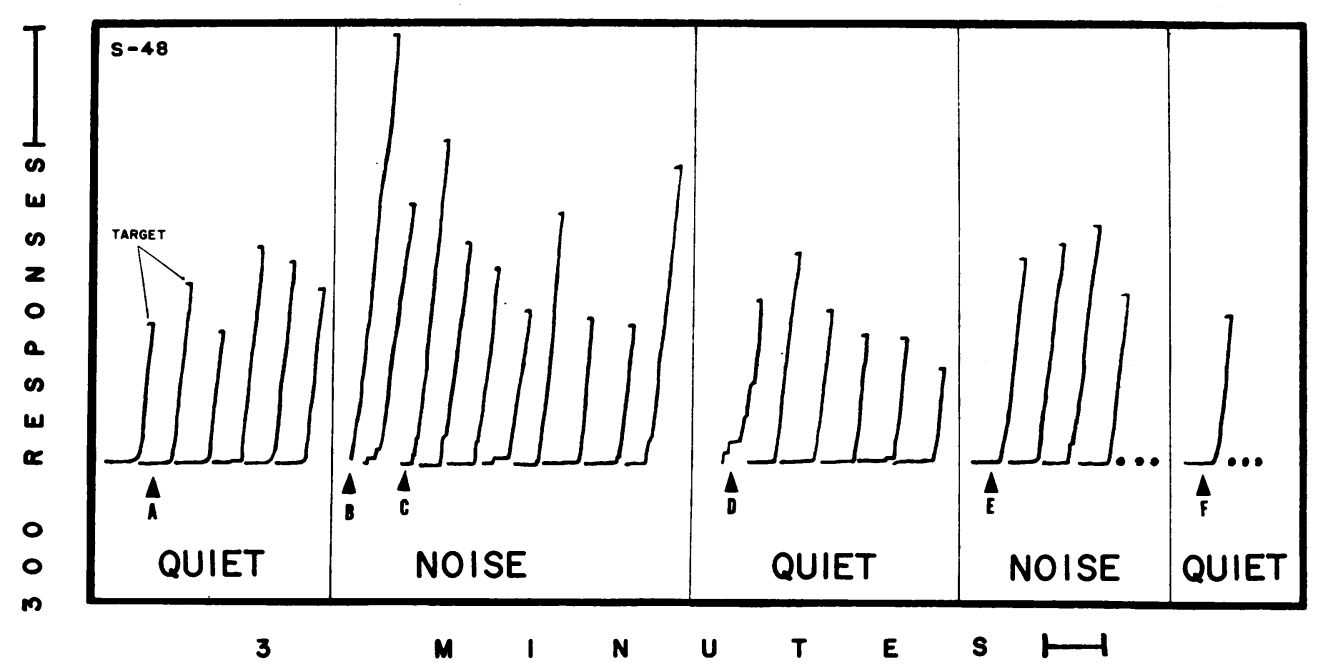

Fig. 1. The effect of alternating periods of intense noise and quiet on the observing responses maintained by a 3 -minute, fixed-interval schedule of target presentation. Each curve is the cumulative record of the observing responses for the 3-minute interval between target presentations. The horizontal pip at the top of each curve designates the target presentation.

At $\mathrm{D}$ the noise was discontinued for 18 minutes. The immediate effect was a burst of responses. During the succeeding 3-minute intervals during QUIET, however, recovery was complete, as evidenced by the absence of responses soon after reinforcement. This "off-effect"was generally briefer and smaller than the previous "on-effect" for all ten $\underline{S}$ 's, and was virtually absent for one $\underline{S}$.

At $\mathrm{E}$ the noise was again introduced. It now produced no change in performance. Similarly, when the noise was again discontinued, at $F$, performance was not changed. Only two $S^{\prime}$ s showed any disruption during the second presentation of the same intense noise.

The changes observed in Fig. 1 were typical of those found with all ten subjects exposed to noncontingent, intense ( $95-110$ decibels) noise. For each subject the initial onset or termination of the intense noise temporarily disrupted the established temporal discrimination. Repeated presentations and terminations of the same highintensity noise tended to produce progressively diminishing disruption. These results were obtained regardless of whether the noise used was a steady white noise as in Fig. 1), a steady 500-cycle-per-second pure tone (one subject), or an arhythmic, high-pitched, machine-shop type of noise (two subjects). Nor did the results differ noticeably when the machine-shop noise was made sporadic: on for 0.25 second, off for 0.25 second (two subjects).

\section{Target-detection time}

It will be recalled that the target was presented automatically every 3 minutes. The interval between presentation of the target and the release of the switch by the subject defines target-detection time. The detection times of the subjects were recorded, but there were only negligible differences between quiet and noise periods. This absence of difference agrees with the observation from Fig. 1 that the 
rate of observing responses immediately prior to reinforcement (target presentation) did not relate systematically to noise. An incidental difference between noise and quiet might bear mention. During the quiet periods (55 decibels), in eleven instances a subject "dozed off," thereby producing target-detection times ranging from 15 seconds to 1 hour. No such instances of "dozing off" occurred during any of the noise-presentations.

Variations in Noncontingent Noise

The temporary disruptions obtained in Fig. 1 upon the introduction or termination of an intense noise were also obtained upon the introduction or termination of noises that were much less intense. Figure 2 presents the effects of several variations of noise-presentation.

In Fig. 2A, a considerably reduced intensity ( 85 decibels) produces a disruption of the temporal discrimination at the initial introduction and removal of the noise, with recovery occurring by the fifth reinforcement. In Fig. 2B, an even lower intensity-- in the form of a hum-- was introduced for only 5 seconds. This hum was simply loud-speaker background noise and its intensity was only 60 decibels. Nevertheless, this brief audible hum was sufficient to produce a conspicuous disruption in temporal discrimination as evidenced by the increase in observing responses for about 30 seconds. For the figures presented up to now, the initial period was one of quiet, with noise introduced later. In Fig. 2C, however, initial conditioning occurred with $\mathbf{7 0}$ decibels of white noise continuously present. The noise was then discontinued and the result was again a disruption in temporal discrimination immediately following termination of the noise.

Figure 2D presents the results of repeated presentations of different types of noise. The initial presentation of 80 decibels, the initial onset of quiet, and the initial presentation of a hum each in turn produced a reduction in pause. However, the second presentation of 80 decibels and the second onset of quiet produced no change. Finally, even the initial presentation of music at 90 decibels produced no change. Figure 2E shows the effects of several other types of successive changes in the acoustic pattern, without an intervening quiet period. The initial presentation of a barely discernable loud-speaker hum, the onset of quiet, and the initial presentation of 75 decibels each in turn produced a disruption with rapid recovery. When the 95-decibel presentation followed the 75-decibel presentation (without a period of quiet intervening), a disruption was again produced. A quiet period produced only a small burst of responses. When the 75 decibels and 95 decibels were again presented, no change in performance occurred, and the same was true of the third introduction of a quiet period.

The data of Fig. 2 clearly indicate that the prerequisite for the disruption of the temporal discrimination seen in Fig. 1 is not the occurrence of a high intensity, but simply the occurrence of change in intensity. An increase of noise, a decrease of noise, the introduction of quiet, or a change of the noise pattern all disrupted the temporal discrimination at the moment of change, with recovery occurring within the next 15 minutes. Successive presentations of the same intensity produced little or no behavioral change, but successive presentations of different intensities produced successive disruptions. Finally, a point was reached where even the initial introduction of a new intensity or pattern produced no behavioral change.

The introduction of acoustic change seems to have caused the performance to revert temporarily to its unconditioned pattern. The disruption of fixed-interval 


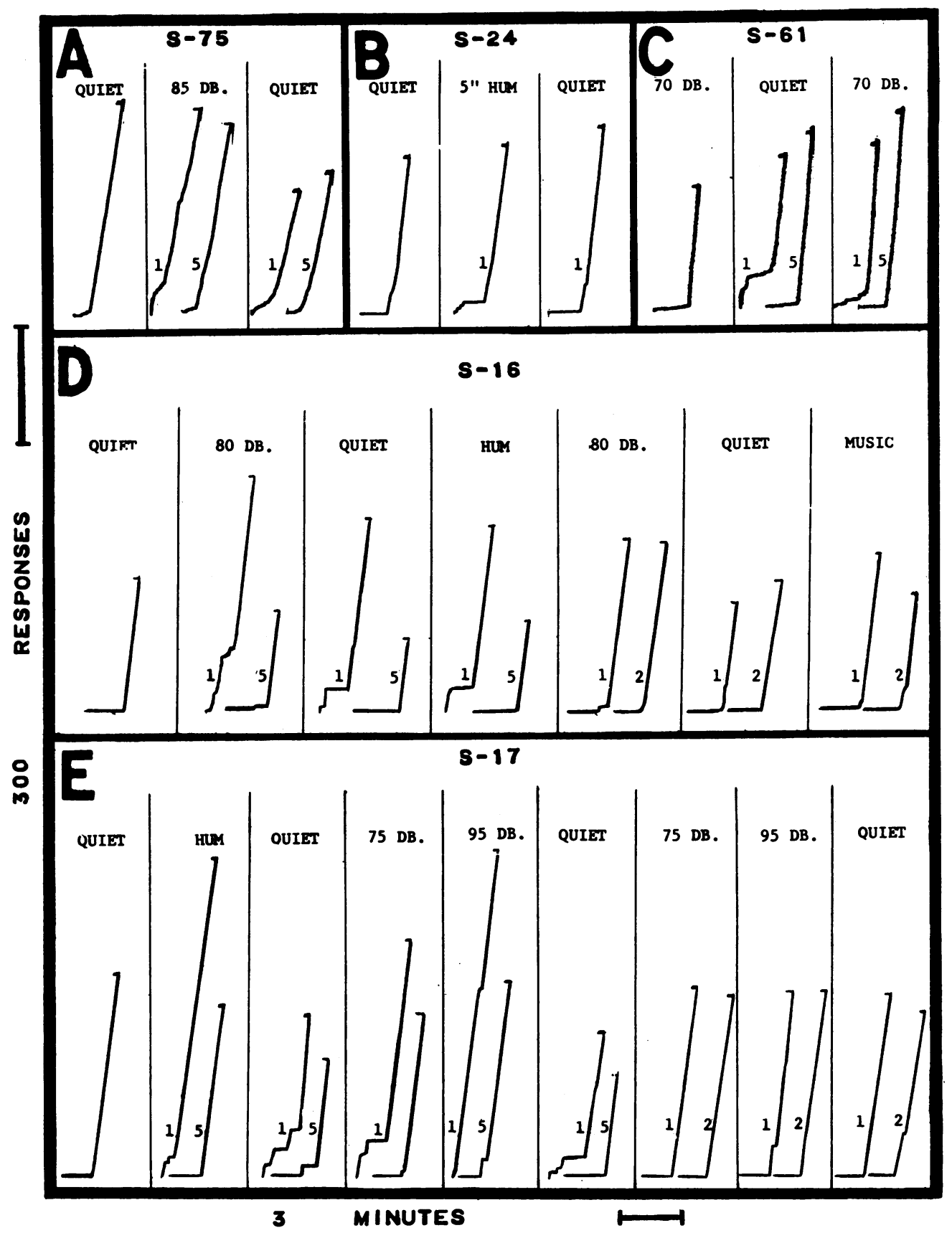

Fig. 2. The effects of noncontingent noise on observing responses during a 3-minute, fixed-interval schedule of target presentation. The initial curve for each subject represents stable fixed-interval performance. The numerals 1,2 , or 5 adjacent to a given curve designate whether that curve was the 1st, 2nd, or 5 th curve obtained under the specified noise condition. The various noise intensities were presented in left-to-right-sequence. 
performance following the introduction of novel stimuli has been established by Ferster and Skinner (7), who used visual stimuli with pigeons. The findings of this and many of the previous experiments on the effects of noncontingent noise can be accounted for on the basis of stimulus change. Various other explanations have been proposed to account for the effects or lack of effects of noncontingent noise, especially in terms of competing responses, fatigue, or some inner compensatory mechanism.

An explanation of the present findings in terms of competing responses assumes, minimally, that these competing responses should exist for at least the duration of the noise which produced them. If any change in the strength of these competing responses were to be expected, it would be in the direction of a progressive increase during continued exposure to noise. Termination of the noise should, therefore, reasonably lead to elimination of these competing responses. The present findings seem to make this account untenable since no maintained or progressive increase in the disruption occurred. Instead of a disruption (competing responses) to be eliminated by the termination of the noise, the disruption was produced by it. Explanations in terms of inner fatigue suffer from the same difficulties, since fatigue should be cumulative with continued exposure to the fatiguing conditions. Explanations in terms of some compensatory mechanism have long been used(21) to account for the excellence of performance during exposure to intense noise. In the past this explanation suffered from the difficulty that the hypothesized was unobservable. Presumably, the subject makes a greater effort via increased attentiveness and thereby maintains his level of performance. Insofar as the Holland Procedure used in this investigation provided a direct measure of attentiveness (observing responses), no such continued compensatory effort occurred. Why there should be a need for competing against noise intensities of 60 decibels, or against silence (off-effect), is difficult to understand. An account of the observed performance changes in terms of stimulus change would seem to be more simple, direct, and parsimonious.

\section{EXPERIMENT II: NOISE AS A DISCRIMINATIVE STIMULUS}

The procedure followed here was the same as that in the previous experiment except that noise was now delivered in a specified temporal relation to the target. A 110-decibel white noise was continuously present but was discontinued for the 15 seconds preceding each target. Figure 3A shows that under these circumstances the responses occurred only during the 15-second quiet period immediately preceding each target and never during the initial 165 seconds of noise. The temporal discrimination seems to be greatly improved. In Fig. 3B the temporal relations were reversed such that the noise was delivered during the last 15-second period rather than the initial part of the 3-minute interval. The first curve of Fig. 3B shows a marked change in performance following this reversal. Responding occurred during the entire initial part of the interval and ceased abruptly as soon as the noise was presented (arrow) at the end of the interval. No further responses were made during the noise for about 60 seconds, even though the target was now presented and could have been detected if an observing response had been made. It would seem that the absence of noise had assumed complete control over the observing response in Fig. 3A, to the almost complete exclusion of the control otherwise exerted by the purely temporal properties of the fixed-interval schedule. Recovery occurred during the presentations following Curve 1 of Fig. 3B, so that by the 14th interval no responding occurred during the initial part of the interval. Responding now seemed to come under the stimulus control of noise, a reversal of the situation obtained in Fig. 3A. The temporal relation of the noise to the target was again reversed in Fig. 3A1. Responses again were made during the early part 
of the 3-minute interval following the reversal, but the stimulus control was not so complete here, however, and the target was detected shortly after its presen-

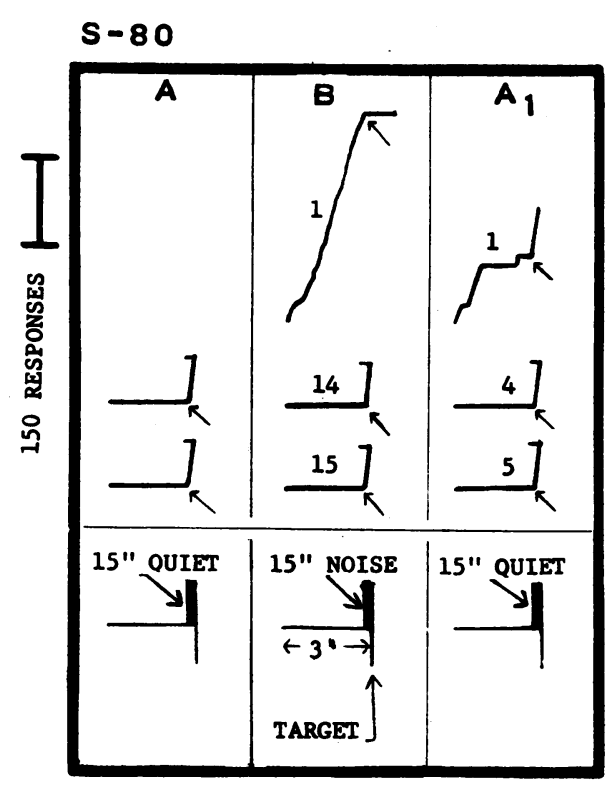

Fig. 3. Noise as a discriminative stimulus. Observing responses are being reinforced according to a 3-minute, fixedinterval schedule of target presentation. Noise, or its absence, occurs for the 15 seconds (arrows) preceding the target. The numerals (n) adjacent to a curve specify that curve as the nth during the designated noise condition. tation. The succeeding intervals showed complete recovery and reinstatements of the effects obtained in Fig. 3A.

Previous animal studies have also demonstrated improved temporal discrimination when visual discriminative stimuli were added to a fixed-interval schedule (26). Similarly, reversal of the discriminative stimuli in these studies had also produced a reversal of the usual temporal discrimination. These results indicate that the subject came to "rely upon the noise," with little regard for the purely temporal factors of the reinforcement schedule. The effect of the noise-presentation, prior to its reversal, was to improve performance and not to disrupt it.

\section{EXPERIMENT III: NOISE AS} AN AVERSIVE STIMULUS

\section{Noise Escape}

In this experiment, the presence or absence of noise was determined by the occurrence of responses. Consider first the condition in which the responses were followed by the immediate termination of noise (noise-escape). Figure 4 presents the cumulative records of the responses of one subject while he was exposed suc-

cessively and without interruption to quiet, to continuous noncontingent white noise at 95 decibels, and then to noise-escape at the same intensity.

When the continuous noise was introduced at $\mathbf{A}$, the well-developed temporal discrimination seen during the previous quiet period was disrupted temporarily, but recovered almost completely by $B$. Both the number of responses and the temporal discrimination remained unaffected for the remainder of the presentation of the continuous noise (cf., Fig. 1 and 2). At $\underline{C}$ the conditions were changed such that each response in the presence of noise terminated it for 5 seconds. Furthermore, each response in the absence of noise postponed it for 5 seconds. The experimental conditions can be described as a 3-minute, fixed-interval schedule of reinforcement concurrent with a noise-escape schedule having a response-noise interval of 5 seconds and a noise-noise interval of 0 second.

A low steady rate of responses within the first 2 minutes after reinforcement could be seen occasionally, as at $D$, following the addition of the noise-escape contingency. This low rate changed to a moderate steady rate at $\mathrm{E}$, which was maintained for the remainder of the session. The pattern of responding consisted of two components: the first was a moderate rate following each reinforcement ${ }_{\hat{O}}$ changing abruptly (as at F) to the high terminal rate, which was the second component. 


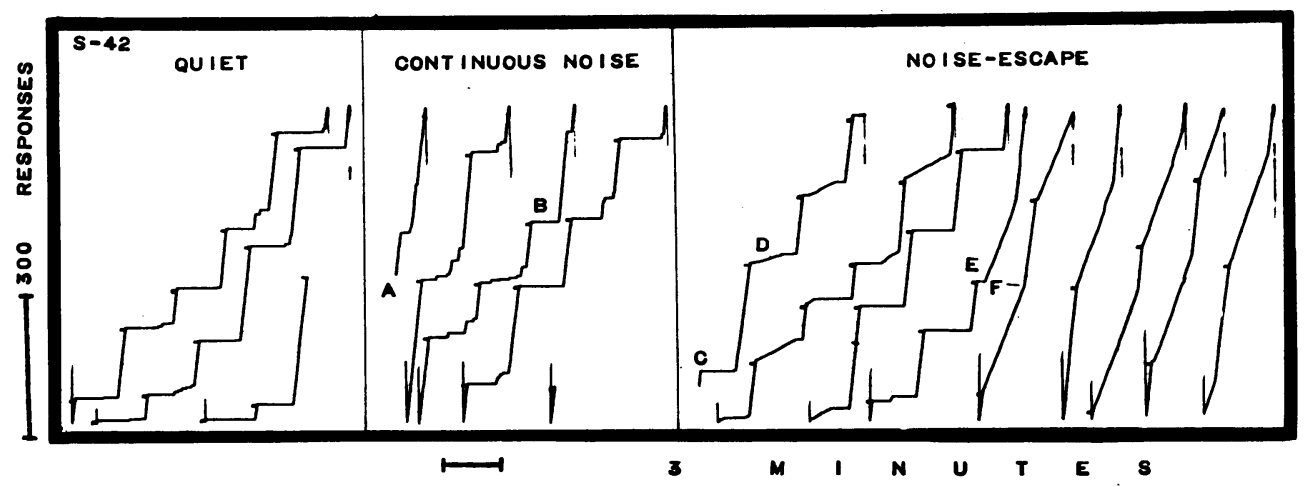

Fig. 4. A comparison of noise-escape and continuous noise. Observing responses are reinforced according to a 3-minute, fixed-interval schedule of target presentation. The actual delivery of noise is not indicated by the recording pen during the response-escape procedure, but can be presumed to have occurred whenever a duration greater than 5 seconds elapsed without a response. Target presentation is indicated by the horizontal pip of the recording pen. The pen resets at the top of the recording paper and continues on at the bottom.

The first component has been produced by the addition of the noise-escape contingency and resulted in the complete elimination of noise from $\mathbf{E}$ to the end of the session. The second component was produced by the target presentation and represents no change of the previous performance. The stable modification of behavior produced by the noise-escape procedure is in marked contrast with the transient changes produced by the preceding continuous noise. It may be noted that the rapid recovery during continuous noise could not be entirely due to sensory adaptation since the same noise intensity produced stable changes in performance during the noise-escape schedule. The effects of a similar concurrent schedule have been studied previously, with rats, using shock as the aversive stimulus, and the results are similar to those seen here (7).

The aversive effects of noise could be seen even more clearly when the responseescape contingency was alternated with target intervals during which no noise was scheduled. (See Fig. 5.) The target was presented as usual every 3 minutes; but every other 3-minute interval contained the provision that 105-decibel noise was terminated or postponed for 5 seconds following each response. The result was an alternation between the usual fixed-interval pattern of responding under no noise ( $A_{1}$ and $A_{2}$ ) and the two-component performance under the noise-escape contingency (B1 and $\left.B_{2}\right)$. This alternation was maintained with only one actual delivery of noise, at $\mathbf{C}$. The noise apparatus was disconnected, at $\underline{D}$, but the responses continued on in the same complex alternating pattern for half an hour before the escape component extinguished. (See $\underline{E}$ and $\underline{F}$.

The two-component pattern of responding was produced by the addition of the noise-escape contingency with all but one of the ten subjects who were exposed to this procedure. Figure 6 shows the cumulative record of the responses of this one exception. The fixed-interval pattern of responding was fairly typical from the beginning of the record to the time that the noise-escape contingency ( 110 decibels, R-N: 5 seconds) was added, at A. For the five intervals following the addition of 


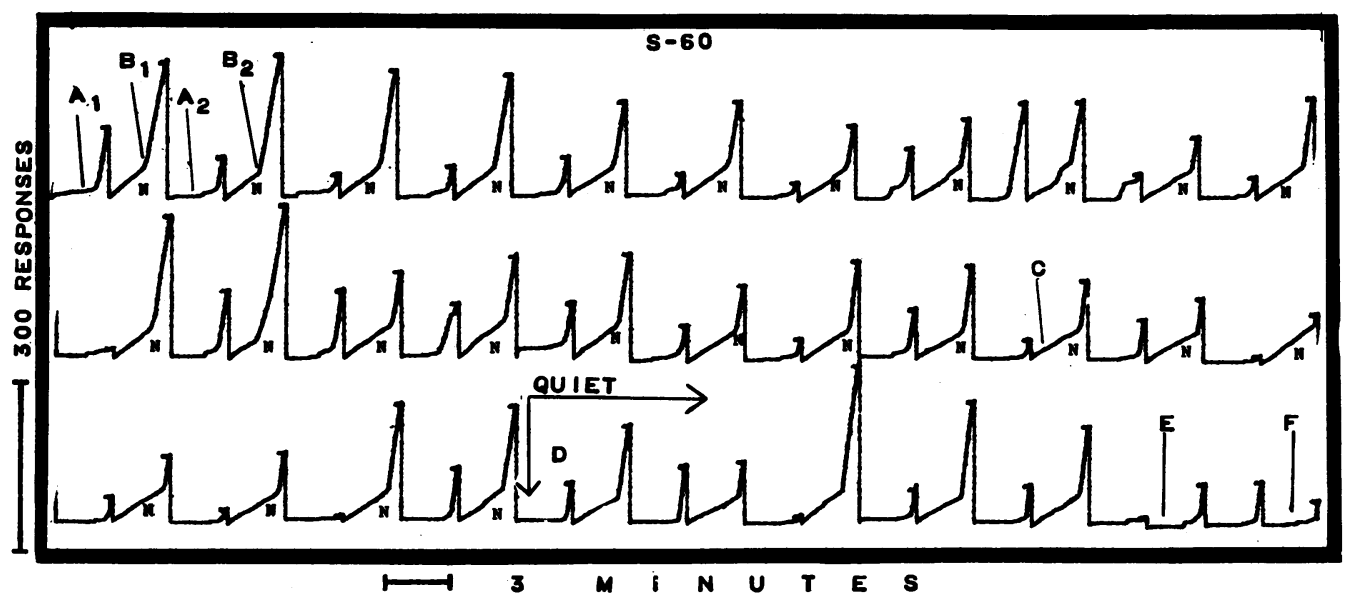

Fig. 5. A 3-minute, FI schedule of target presentation with a noise-escape contingency ( $R-N$ : 5 seconds, $N-N$ : 0 second) added to every other 3-minute interval (designated as $\mathrm{N}$ ). The recording pen resets after every target.

\section{5-63}

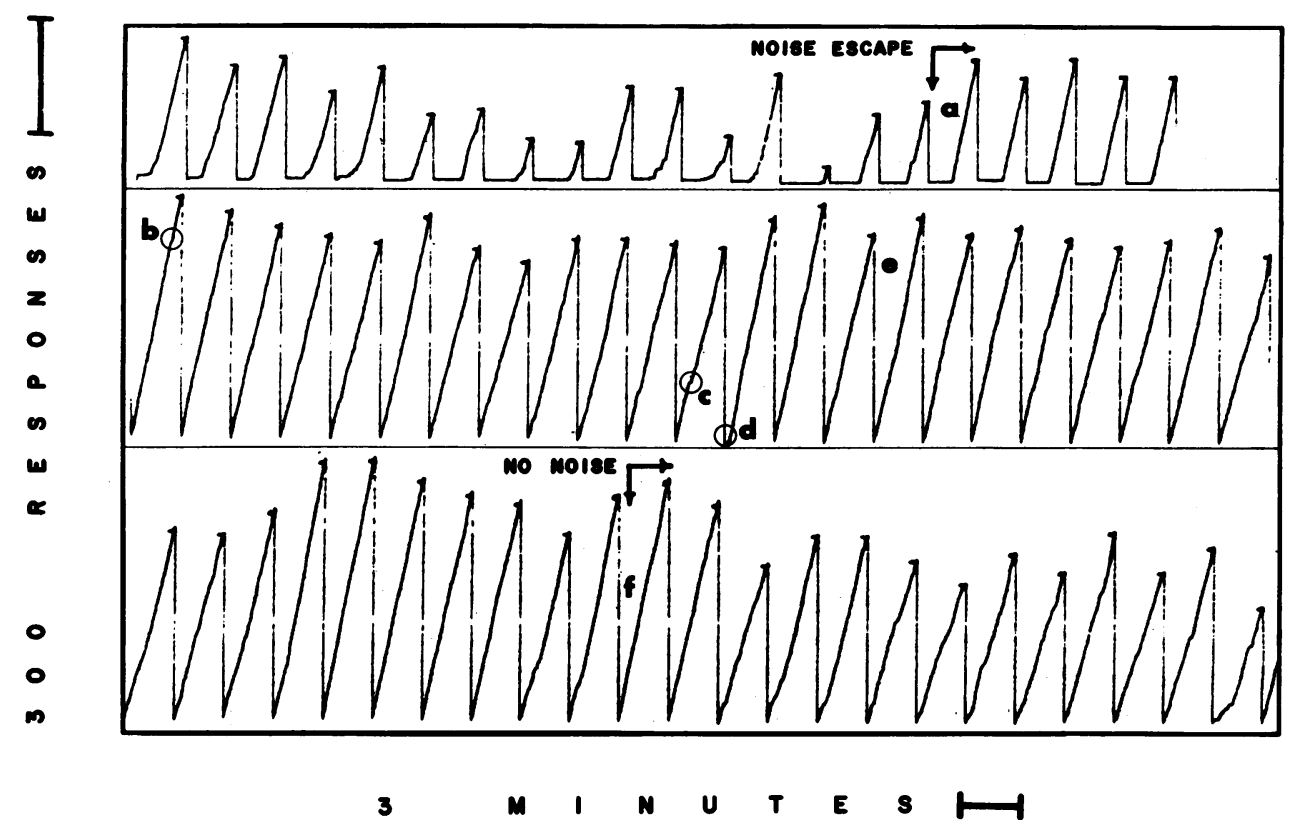

Fig. 6. Exaggerated avoidance responding during a concurrent 3-minute, fixedinterval and noise-escape schedule. 
the noise-escape contingency, no responses were made during the initial part of these intervals. It may be noted that the responding during the latter part of these intervals occurred at a high steady rate, with no rates intermediate between that rate and zero. Noise, therefore, was presented continuously during the periods of zero rate, and no noise was present during the high rates. At the 6th interval following the addition of the noise-escape contingency, responding occurred at a high rate throughout the interval (2nd row of Fig. 6) with only a slight pause at $b$. Because of the high rate, only three bursts of noise were actually delivered, at $\bar{b}$, $c$, and $d$. The response-noise interval was increased, at e, from 5 seconds to $\overline{\mathbf{1 5}}$ seconds, but this increase had no effect since no further pauses appeared. Even when the noise apparatus was disconnected, at $f$, responding continued at a high rate for the remaining $\mathbf{4 0}$ minutes with only a slight decrease.

The high rate of avoidance responses with few deliveries of the noise resembles the high resistance to extinction reported by Solomon, Kamin, and Wynne (27), and by Sidman (24). This absence of a lower and more appropriate rate of avoidance behavior may represent the usual high rate found in the early stages of avoidance conditioning (23). The failure of the high rate to decrease over the 2-hour period makes it more likely that there was some "superstitious" reinforcement of the prevailing high fixed-interval rates by the noise. After the noise-escape schedule was introduced, responding somewhat fortuitously occurred only at a high rate or at a zero rate, but at none of the intermediate rates that are usually seen. Unless intermediate rates did occur, such rates could not be differentially reinforced by the absence of noise and the reinforced (no-noise) high rates would be expected to prevail for some time. O. Lindsley has observed a similar effect with adult humans during an analagous concurrent schedule involving noise (personal communication).

\section{Noise Intensity and $\mathbf{R}-\mathrm{N}$ Interval}

In a study of the determinants of the noise-escape behavior, the observing responses may be superfluous and can be eliminated by turning on the overhead lights, thereby obviating the necessity for their existence. Figure 7 presents the cumulative record of the responses of one subject during which time the overhead illumination, the noise intensity, and the response-noise interval were changed. $A$

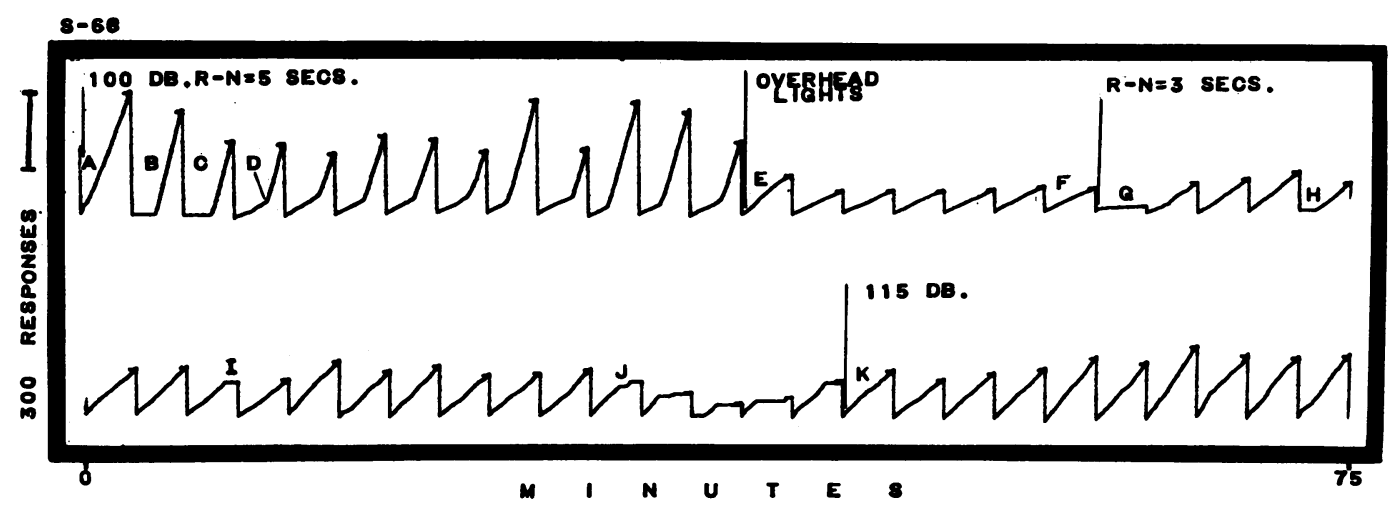

Fig. 7. Effects of a decrease of the $R-N$ interval and an increase of noise intensity upon noise-escape behavior. The recording pen resets after each presentation of the target. 
noise-escape contingency ( $R-N$ : 5 seconds, 100 decibels) was added to the existing fixed-interval schedule of target presentation at the beginning of the record. After a brief transition period, $\underline{A}, \underline{B}$, and $\underline{C}$, this concurrent schedule produced the usual two-component performance, at $D$. The overhead lights were turned on, at $E$, and the target needle could then be clearly seen without the necessity of the observing responses. Almost immediate extinction of the observing responses took place, leaving only the low steady rate of escape responses, from $\mathbf{E}$ to $F$. When the $R-N$ interval was reduced from 5 seconds to 3 seconds, at $G$, the pattern of responding changed in a complex manner. The over-all rate of response with the $R-N$ of 3 seconds ( $G$ to $K$ ) was higher than with the $R-N$ of 5 seconds ( $E$ to $G$ ). But the frequent appearance of pauses, as at $H, I$, and $J$ to $K$, reduced this difference in over-all rate and permitted the frequent delivery of noise. The local rates during the reduced $R-N$ interval, however, were consistently greater than the local rate during the 5-second $R-N$ interval. The reduction of the $R-N$ interval seems to have greatly increased the local rate of responding and the frequency of noise-delivery, but only slightly increased the over-all rate of responding because of the longer pauses. When the noise intensity was increased from 100 decibels to 115 decibels, at $\mathbf{K}$, the over-all rate increased; but this general increase was due primarily to the elimination of the long pauses, the local rate remaining about the same.

The changes following the reduction of the $R-N$ interval are consistent with the findings of Sidman (22), with rats on a shock-escape schedule, that decreases of the $R-S$ interval (analogous to the $R-N$ interval here) produce an increase of the number of shocks as well as an increase of the over-all rate of responding. Barry and Harrison (3), using a noise-escape procedure with cats somewhat similar to that used here, also found that the usual increase in over-all rate at higher noise intensities was largely a function of the elimination of long pauses.

\section{Escape v8. Avoidance Behavior}

During a noise-escape schedule, two different types of response patterns can theoretically emerge. The first is the avoidance-type, in which the responses are made in the absence of noise; the second is the escape-type, in which responses are made in the presence of noise. Rarely did these two types of response patterns exist in pure form, but the extent to which one or the other patterns predominated was found to produce marked differences in the speed of extinction. Examples of predominantly avoidance-type responding were seen in Fig. 4-6, in each of which very few presentations of noise resulted. Behavior was seen to change quite gradu-

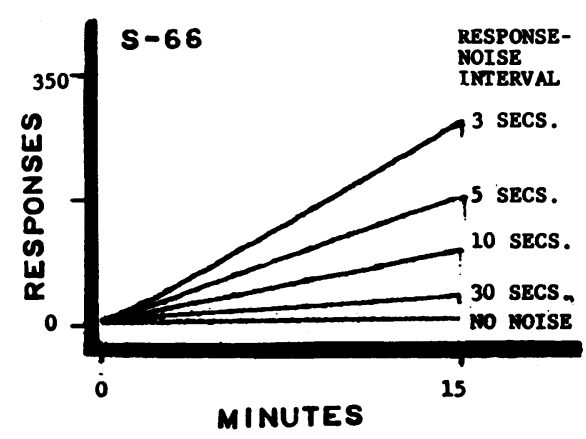

Fig. 8. Changes in responding as a function of the response-noise interval during noise-escape conditioning. ally when the R-N interval was increased (Fig. 5) or when the noise was experimentally disconnected (Fig. 4 and 5).

Figure 8 presents segments of the cumulative records of the responses of one subject who showed a fairly pure case of the escape-type pattern of responding. In this special case, a single response was usually made almost immediately following the onset of noise, with no additional responses during the resulting quiet period even though such responses would have postponed the noise for an additional period. (The overhead lights were on so that the usual observing responses were not 
made.) The duration of the $R-N$ interval was varied in a scrambled sequence from 3 - 30 seconds, with 15 minutes of exposure to each of the R-N values. Each change of the R-N interval was made at " 0 " minute. It can be seen that the rate of response changed immediately with each change of the R-N interval, and no further changes in rate occurred during the 15-minute exposure. Immediate and complete extinction took place when the noise was experimentally disconnected. Unlike the avoidancetype of response pattern, the escape-type is accompanied by frequent presentation of the aversive stimulus. Therefore, changes in the scheduling of the aversive stimulus seem to affect behavior within a very short time.

The noise intensity was increased from 105 decibels to 117 decibels during a second exposure to the R-N interval of 5 seconds, in an attempt (not shown) to modify the escape-type of pattern. The escape-type of response pattern remained, however, and the total number of responses was increased only slightly (by 5 responses) during the 15-minute period. No other evidence was obtained as to the emergence of one pattern over the other.

\section{Noncontingent Noise Following Noise-escape}

When noncontingent noise was presented following conditioning on a noise-escape schedule, the responses extinguished but not smoothly. Figure 9 presents cumulative records of the responses of one subject who had received extensive prior conditioning with various noise intensities and R-N intervals. Although the target was still being presented, the overhead lights were on and there were, therefore, no observing responses. From the beginning of the record to $\mathrm{A}, 80$-decibel noise with an R-N interval of 5 seconds was scheduled. The result was the usual low rate of escape responses. The escape contingency was remored, at $A$, and the noise was delivered continuously for the remainder of the session. Responding ultimately was reduced to zero, but the immediate effect of the continuous noise was to produce a series of alternating pauses and bursts (especially at B) which had a local rate higher than that ever seen during the noise-escape schedule. These bursts seem similar in many respects to the bursts produced in animal studies by brief, noncontingent shocks following avoidance conditioning (25).

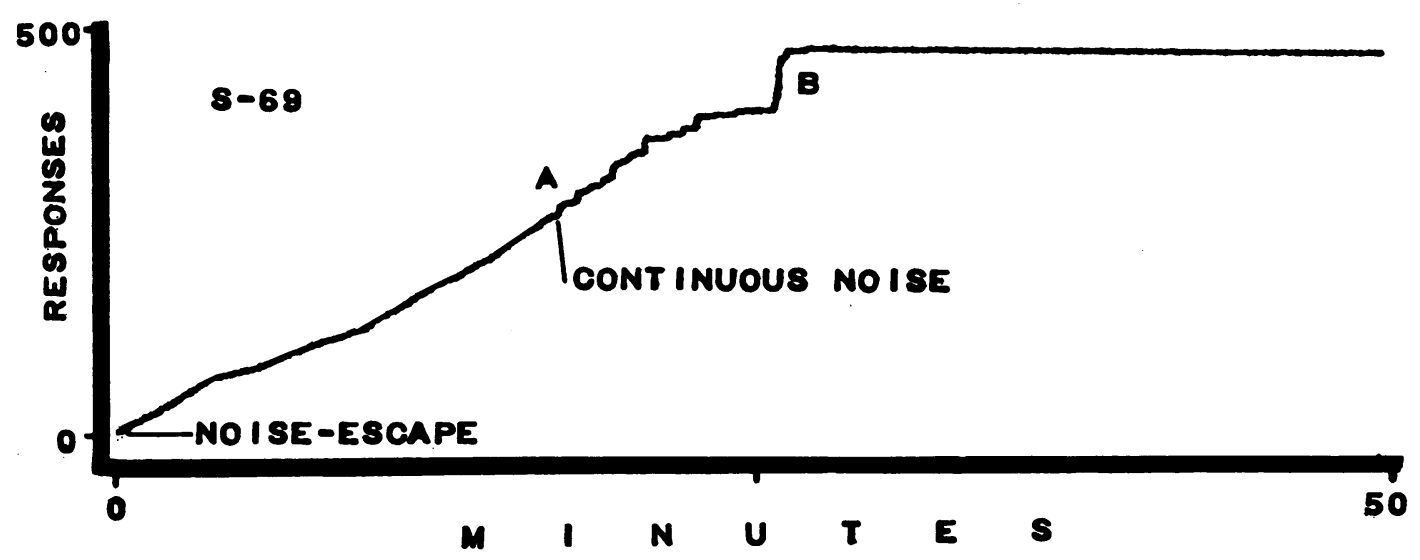

Fig. 9. Cumulative record of the responses during a noise-escape schedule followed by the delivery of noncontingent noise. 
The presentation of the continuous noise seems to have reinstated the stimulus conditions under which responses had been reinforced previously by the termination of noise. The responses, therefore, occurred at a high rate (burst), which would be expected to extinguish (pause) since the noise was not terminated. This absence of responding and the simultaneous presence of noise would again reinstate the stimulus conditions under which responses had been reinforced, and responding again would be expected to occur at a high rate. The result would be the observed series of pauses and bursts which would be expected to extinguish eventually for lack of differential reinforcement.

The complete extinction of responding during the continuous noise provides additional support to the findings of Experiment I concerning the slight effects of such a method of noise-presentation.

\section{Response-produced Nolse}

Intense noise can be used to eliminate behavior if it is delivered immediately following the occurrence of that behavior. Figure 10 illustrates the typical changes which took place for six subjects when intense noise was delivered for 5 seconds following each observing response during the 3-minute, fixed-interval schedule of target presentation. No noise was delivered from the beginning of the record to $C$, and the responding curing that time often showed a smooth acceleration, as at $\bar{A}$ and $B$. The introduction of the response-produced noise ( 110 decibels) produced an initial increase of responding followed by a decrease for the remainder of the noisepresentation. This decrease was not general, however; rather, the low rates such as had been seen previously, at $\mathbf{A}$ and $B$, were selectively eliminated and the high terminal rates were usually unchanged, as at $D$. Within each 3-minute interval, responding was postponed to the end of the interval, occasionally to such an extent that the target remained undetected for some time after its presentation, as at $\underline{E}$ and $F$. The temporal discrimination was generally improved, therefore, but at the expense of rapid target detection. When the noise apparatus was experimentally disconnected, at $\mathbf{G}$, recovèry was typically gradual. The low rates and smooth acceleration returned in a few instances, as at $\mathrm{H}$ and $\mathrm{I}$, but the improvement of the temporal discrimination persisted for some time, as at $J$ and $K$. It would seem that once the noise had forced the responses into a more efficient temporal pattern, this improvement lingered on after the cessation of the noise.

The effect of response-produced noise was seen even more clearly when the noise was presented during a fixed-ratio schedule of target presentation for one subject. (See Fig. 11.) Every 20th observing response produced the target after previous conditioning at smaller ratios. At the start of Fig. 11, no noise was delivered and responding showed the usual fixed-ratio pattern. When 110-decibel noise was introduced at $\mathbf{A}$, for 5 seconds following each response, responding was almost completely eliminated and did not return for several minutes after the noise had been disconnected, at $B$. Once responding did occur, it immediately resumed the characteristic fixed-ratio pattern for the remainder of the session.

In Experiment I, it was found that the changes in performance due to noncontingent noise were largely independent of noise intensity. The same would appear to be true when noise is used as a discriminative stimulus (Experiment II). The response-contingent noise of Experiment III, however, produced stable performance changes only at fairly high intensities. During the initial stages of investigation, the noise intensities used were usually below 105 decibels and the responses usually 


\section{S-56}

1
1
0
1
0
2
0
0
$\infty$
1
0
0
0

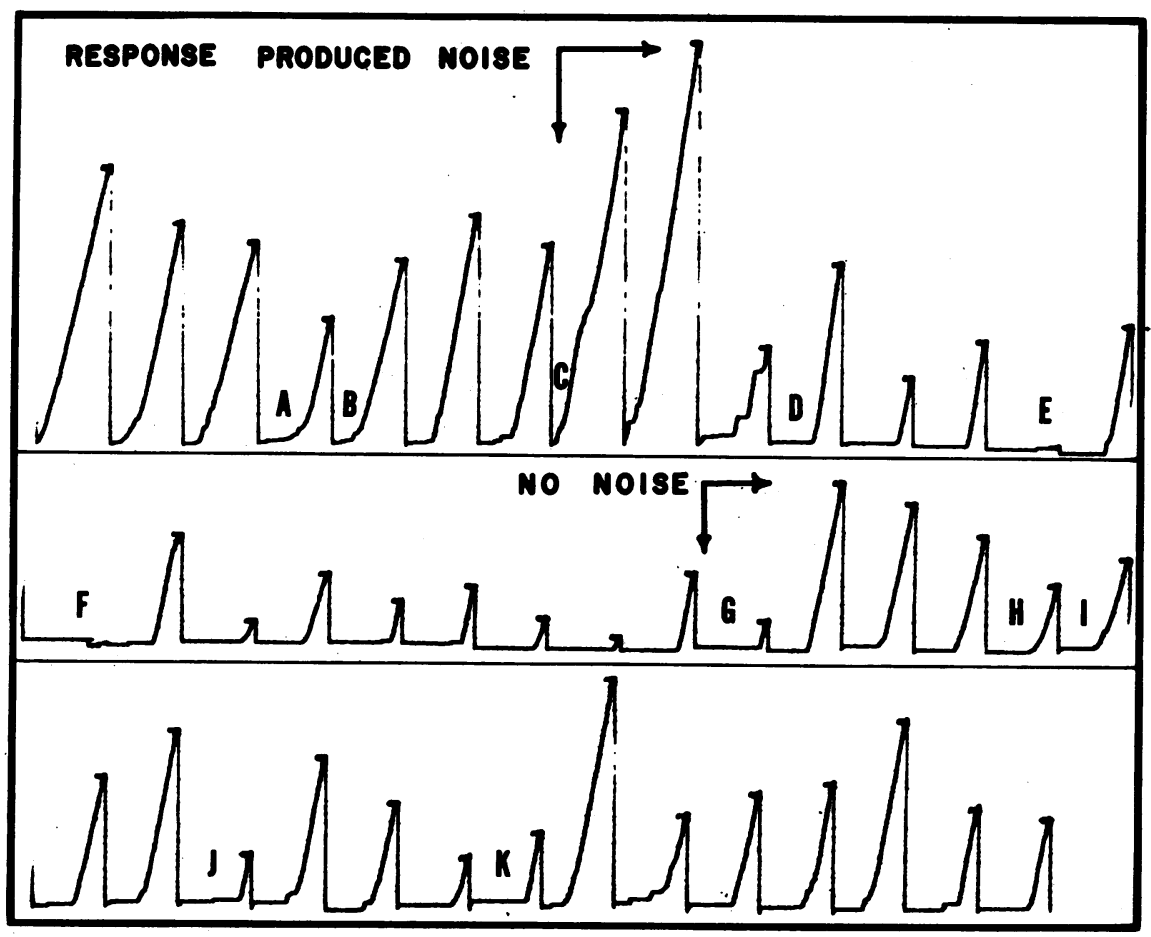

3

$\begin{array}{lllllll}M & \boldsymbol{I} & \boldsymbol{N} & \boldsymbol{U} & \mathbf{T} & \mathrm{E} & \mathrm{S}\end{array}$

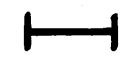

Fig. 10. The effect of response-produced noise upon the observing responses maintained by a 3-minute, FI schedule of target presentation.

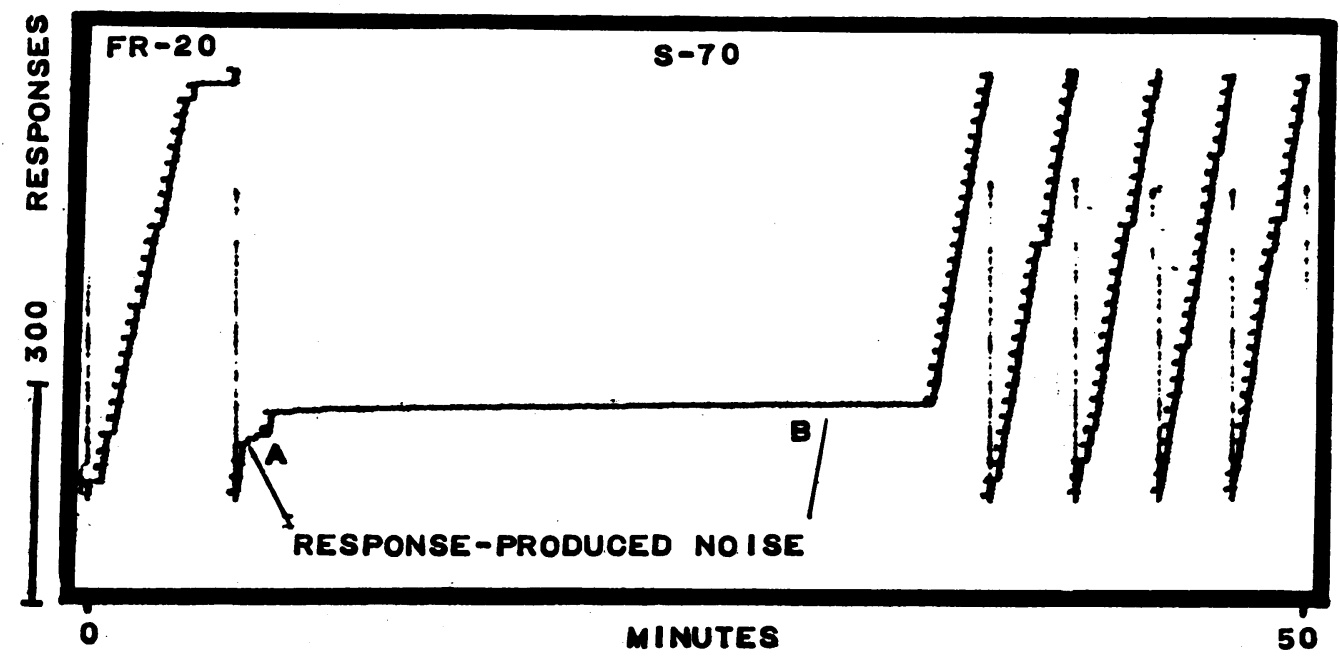

Fig. 11. Effects of response-produced noise on the observing responses maintained by a fixed-ratio schedule of target presentation. 
produced (or eliminated) the noise for durations less than 1 second. Under these moderate conditions of noise-presentation, only 7 out of $18 \underline{S}^{\prime} s$ showed stable performance changes during the noise-escape schedules, and only 2 out of 16 during response-produced noise. When intensities of 105-120 decibels and durations of 3-5 seconds were used, 2 out of 12 S's showed stable performance changes during response-escape, and 7 out of 7 during response-produced noise. These results refer to the initial noise intensity presented to a given $\underline{\mathbf{S}}$.

For several $S^{\prime} s$, the intensity was reduced once a stable change in performance occurred. Under these circumstances, fairly low intensities maintained the changed performance for some time. In Fig. 9, for example, the initial conditioning took place with 115 decibels, after which it can be seen that only 80 decibels was sufficient to maintain the escape responses. Such a low intensity was never observed to produce stable performance changes during initial escape conditioning.

\section{CONCLUSIONS}

The present investigation has shown that intense noise can serve as an aversive stimulus to modify human behavior. It has been known that an aversive stimulus must be delivered contingent on behavior if it is to be maximally effective. For example, when shock has been delivered independently of behavior, the effect on the ongoing behavior was found to be trivial compared with the changes produced by response-contingent shock (1). Similarly, the present investigation found that the major changes produced by noncontingent noise were transient in duration. Yet, previous studies of the effects of intense noise on human behavior have been almost exclusively restricted to the use of noncontingent noise. Under these circumstances, noise has been found to produce little or no behavioral change. The present investigation indicates that many of the behavioral changes attributable to high-intensity noise in past studies may have been largely due to stimulus change.

Noise also was found to produce stable behavioral changes when it was used as a discriminative stimulus. As in the case of noncontingent noise, this type of control was not related to the aversive properties of intense noise. Rather, this discriminative control can be exerted by stimuli of low, as well as high, intensity. When used in this manner, noise is commonly considered to have "meaning," and then seems more likely to be referred to as sound than as noise.

Intense, response-contingent noise was found to produce large and stable modifications of performance, but seemingly noncontingent noise possibly may also alter performance. For example, intense noise may reinforce the frequent absence of a subject from a noisy room, locale, or machine if such an absence produces a reduction of the noise. The subject's over-all work output might very well be reduced by the sheer fact of his absence even though the performance itself did not directly change the noise presentation. This indirect effect may account in part for the reported performance deficits in field studies since such absences are difficult to control under those circumstances. In the present study, for example, an occasional subject left the experimental chamber because of the delivery of "noncontingent" noise. His over-all "work-output" was reduced, of course.

One of the seeming paradoxes concerning the effects of intense nolse has been the ease with which strong subjective statements of amnoyance could be obtained in spite of the insensitivity of performance. These subjective reactions possibly are concerned with the aversive properties of the noise and reflect the subject's tendency to reduce or eliminate it. If this interpretation is correct, no paradox 
need exist since the usual deficits are probably a function of stimulus change, whereas the subjective verbal reports would be a function of the aversive properties.

\section{SUMMARY}

Young adult humans were conditioned according to a fixed-intervai and fixedratio schedule of target presentation, and various conditions of noise were introduced. It was found that the degree of control exerted by noise over behavior was largely a function of whether the noise had any differential relation to the target or to the responses. When noise, or its absence, was used as a discriminative stimulus for the target, responding came under the control of the noise, or its absence. Similarly, when intense noise, or its absence, was made contingent upon responses, the pattern and frequency of responding were found to vary as a function of the conditions of noise presentation. When the noise was not presented in some differential relation to the target or the response, its major effects were transient and largely predictable on the basis of stimulus change.

\section{REFERENCES}

1. Azrin, N. H. Some effects of two intermittent schedules of immediate and non-immediate punishment. J. P s y c hol. . 1956, 42, 3-21.

2. Baker, K. H. Pre-experimental set in distraction experiments. J . g e n . P s y c h ol.. 1937, $16,471-488$.

3. Barry, J. J., Jr., and Harrison. J. M. Relation between stimulus intensity and st rength of escape responding. Ps y chol. Rep., 1957, 3, 3-8.

4. Berrier, F.K. The effects of noise. Ps y chol. Bull. 1946, 43, 141-161.

5. Broadbent, D. E. Some effects of noise on visual performance. Q. J. exp. P s y c h ol.. 1954, 6, 1-5.

6. Ferster, C. B. The use of the free operant in the analysis of behavior. P s y chol. Bull.. 1953, 50. 263-264.

7. Ferster, C. B., and Skinner, B. F. Schedules of reinforcement. New York: Appleton-Century-Crofts, 1957.

8. Ford, A. Attention-automatization: an investigation of the transitional nature of the mind. A m. J. P s y c hol., 1929, 41, 1-33.

9. Freeman, G. L. Changes in tension-pattern and total energy expenditure during adaptation to distracting stimuli. A m. J. P s y c hol"., 1939, 52, 354-360.

10. Hanley, T.D., and Williamson, R.J. The effect of various noise levels on perfornance of three mental tasks. SDC.Report No. $104-2-21$, Dec., 1950.

11. Harmon, F. L. The effects of noise upon certain psychological and physiological processes. Arch. Psychol., No. 147, 1-81.

12. Holland, J.G. Technique for the behavioral analysis of human observing. Sci ence. 125. 1957, 348-350.

13 Hovey, H. B. Effects of general distraction on the higher thought processes. A $m e r$. J . P s y c hol., 1928, 40, 585-591.

14. Jerison, H.J. Effects of noise and fatigue on a complex viglance task. W A DC T e ch R port $57-14$, Jan., 1957.

15. Jerison, H.J. Experiments on vigilance: performance on a simple viglance task in noise and quiet. WADC Tech Report 57-318, June, 1957.

16. Kryter, K. D. The effects of noise on man. J. speech hear. Dis., Monog. Suppl. 1, 1950, 1-26. 
17. Laird, D. A., and Coye, K. Psychological measurements of annoyance as related to pitch and loudness. J. a c ou st. S o c. A m e r., 1929, 1, 158-163.

18. Lewis, D. The effect of noise and vibration on certain psychomotor responses. Civil Aero. Adm., Report No. 8, Jan., 1953.

19. Loeb.M. The effects of intense stimulation on the perception of time. AMRL Report No. 269 , Fort Knox, June, 1957.

20. Miles, W. R. Immediate psychological effects. Chpt. 11 in Benox Report: An exploratory study of the biological effects of noise. U. of Chicago. Dec., 1953.

21. Morgan, J.J. B. The overcoming of distraction and other resistances. A r c h. P s y c hol., No. $135,1916$.

22. Sidman, $M$. Two temporal parameters of the maintenance of avoidance behavior by the white rat. J. comp. phy siol. P s y chol., 1953, 46, 253-261.

23. Sidman, M. The temporal distribution of avoidance responses. J. comp. physiol . P s y c hol., 47, 1954, 399-402.

24. Sidman, M. On the persistence of avoidance behavior. J. abnorm. soc. Psychol., $1955,50,217-220$.

25. Sidman, M., Herrnstein.R. J., and Conrad, D.G. Maintenance of avoidance behavior by unavoidable shocks. J. comp. phy siol. P s y chol., 1957,50,553-557.

26. Skinner, B. F. Some contributions of an experimental analysis of behavior to psychology as . a whole. A m er. Ps y chol., 1953, 8, 69-78.

27. Solomon, R. L., Kamin, L. J., and Wynne, L.C. Traumatic avoidance learning: the outcome of several extinction procedures with dogs. J. a bnorm. S oc. P s y chol.. 1953, 48, 291-302.

28. Stevens, S.S., and others. Part I: The effects of noise and vibration on psychomotor efficiency. Psychol. Lab., Harvard U., OSR D Report No. 274 , Dec., 1941.

29. Tinker, M. A. Intelligence in an intelligence test with an auditory distractor. A m e r. J. P s y c hol . , 1925, 36, 467-468. 